\title{
La incidencia de los registros en la producción de ganado bovino y su importancia para conocer su rentabilidad a lo largo de un ciclo productivo
}

\section{The incidence of records in the production of cattle and their importance to know their profitability throughout a productive cycle}

\author{
MSc. Tulio Alberto Piedra Moreno ${ }^{1}$ \\ piedramorenomvz@hotmail.com \\ PhD. Milton Rafael Maridueña Arroyave ${ }^{2}$ \\ milton.mariduenaa@ug.edu.ec
}

Recibido: 1/04/2019; Aceptado: 1/06/2019

\begin{abstract}
RESUMEN
Uno de los pilares fundamentales en la ganadería es conocer y poder diagnosticar el estado actual de los hotos productivos. A través de los registros ganaderos se puede lograr entender el estado actual y los futuros cambios que se necesiten o se puedan dar. Dentro de los tipos de ganadería (intensiva, extensiva) la intensiva es la más común para la producción de leche, que es donde más se tiene implementado estos registros por la necesidad de conocer el total de producción de cada animal y la fecha probable del parto, factor necesario para saber con cuantos animales se va a contar para la producción de leche. El problema se presenta en las ganaderías extensivas ya que, por motivos como la resistencia al cambio, la implementación de tecnologías, el grado de los productores al administrar los registros, usan ningún tipo de registro, convirtiéndose así en una producción la cual no conoce su rentabilidad.
\end{abstract}

Palabras Clave: registros ganaderos, ganadería intensiva, ganaderías extensivas, rentabilidad

\footnotetext{
1 Servidor Público AGROCALIDAD, Guayaquil, Ecuador

2 Universidad Tecnológica Empresarial de Guayaquil, Guayaquil, Ecuador
}

Revista científica Ciencia y Tecnología Vol 19 No 23 págs. 41-44

http://cienciaytecnologia.uteg.edu.ec 


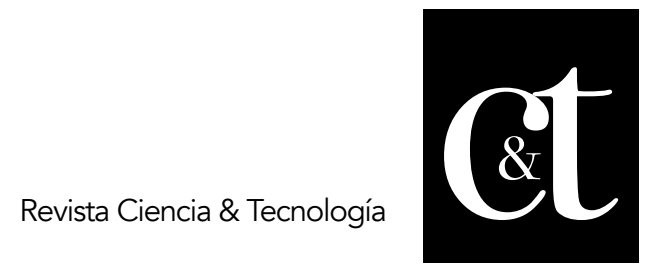

No. 23, 31 de julio de 2019

ISSN impreso: 1390 - 6321

ISSN online: 2661 - 6734

\begin{abstract}
One of the fundamental pillars of livestock is to know and diagnose the current state of the most productive products, through livestock records, you can achieve the current status and future changes that need it. Within the types of livestock (intensive, extensive) the intensive is the most common for milk production, which is where these records have been most implemented by the need to know the total production of each animal and the likely date of birth, necessary factor to know how many animals will be counted for milk production. The problem arises in extensive herds and for various reasons such as resistance to change, the implementation of technologies, the degree of producers to records, farmers who do not use any type of record, thus becoming a production of which no Know your profitability.
\end{abstract}

Keywords: livestock records, intensive livestock, extensive livestock

\title{
Introducción
}

La demanda del mercado de carne y de productos lácteos producidos en forma sustentable crece rápidamente. La producción de bovinos en pasturas o en base a pastos es intrínsicamente sustentable ya que este sistema de producción se basa en la complejidad ecológica y biodiversidad para mantener la producción sin recurrir a inversiones costosas. Los productores de bovinos están comenzando a reconocer que el pastoreo rotacional manejado en forma intensiva (también llamado manejo intensivo del pastoreo o pastoreo planeado) puede disminuir los costos de producción, reducir el estrés animal, y potenciar el sistema inmune de los animales. Esta publicación destaca estas y otras prácticas que los productores están utilizan para proveer a los consumidores de alimento nutritivo proveniente de granjas y ranchos sustentables. (Lee Rhinehart, 2017). En Ecuador existen 4.435.000 según el Proyecto de Erradicación de Fiebre Aftosa 2017 Agrocalidad (Agrocalidad-PEFA, 2017). En la mayoría de fincas ganaderas que se dedican a la producción de leche manejan registros como por ejemplo número de partos, fecha probable de parto, fecha de inseminación, litros producidos diariamente, semanal y mensualmente entre otros. En las ganaderías dedicadas a la producción de ganado de carne los registros principales que se manejan son pesos al nacimiento, peso al desteto, peso la venta, fecha de nacimiento, número de partos, cantidad y tipo de alimento suministrado. Al no tener una herramienta para la toma de sesiones, el ganadero no sabe si su rentabilidad en este negocio es buena o mala; si es conveniente o no seguir con el mismo sistema de crianza (haciendo referencia a la ganancia de peso con la alimentación que estos animales están recibiendo ¿están ganado el peso adecuado? y de no hacerlo tiene que cambiar el tipo de alimentación para mejorar los pesos). Son interrogantes debe de tener muy en cuenta en este negocio. La rentabilidad en la ganadería es un punto a tomar muy en cuenta para mejorar y continuar con esta actividad y sea beneficiosa.

\section{Desarrollo}

La realización de este trabajo es una revisión bibliográfica para analizar la incidencia de los registros en la producción de ganado bovino y su importancia en la rentabilidad a lao largo de un ciclo productivo. (Londoño 2015) en su trabajo de titulación sobre implementación de registros productivos y reproductivos en tres unidades ganaderas 


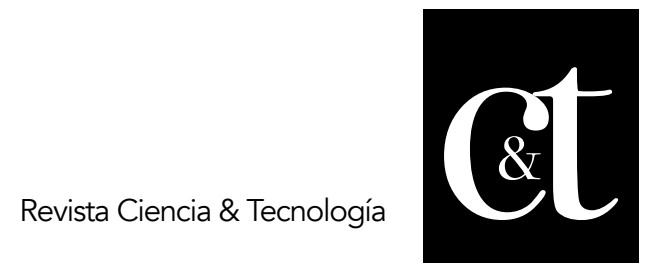

No. 23, 31 de julio de 2019

ISSN impreso: 1390 - 6321

ISSN online: 2661 - 6734

del departamento de Córdoba, indica que el sistema de manejo de finca por computador ha sido adoptado por cerca de 3.000 personas en todo el país, y por lo menos una decena de agremiaciones de productores. Los agricultores y ganaderos colombianos no pueden seguir manejando sus fincas sin una administración ajustada a las condiciones de competencia que existen en la actualidad. Una inversión de 100 millones o más no puede seguir llevando cuentas en papelitos o tarjetas manejadas por el administrador o los obreros. En las empresas ganaderas donde se implementó el manejo de datos sistematizado, se obtuvo las diferencias frente a las que no implementan este manejo, un mayor control de las decisiones que se tomaron a la hora de hacer mucho más rentable la finca. Así también Benavides (2009) en el apartado de resultados y discusión del Informe de Práctica de Especialidad presentado como requisito parcial para optar al grado de Bachillerato en Ingeniería Agropecuaria Administrativa con Énfasis en Empresas Agropecuarias en el literal B. Propuesta de registros contables y programa automatizado pág. 37 indica que el establecimiento de registros en Ganadera Siete Hermanos, se realizó en Microsoft Excel 2007 y el uso de sus aplicaciones como Visual Basic. Con este sistema se logra un ingreso fácil de los datos en los diferentes registros, para obtener resultados positivos en la toma de decisiones. Entendiendo así que el uso de los registros ganaderos es necesario, implementándolos mediante sistemas informativos básicos sencillos, de bajo costo, ayudándonos a poder hacer un análisis de las condiciones del hato ganadero y de la rentabilidad que nos ofrece pudiendo así facilitar la toma de decisiones a futuro.

Del articulo (elnuevodiario.com.ni, 2013) los registros pecuarios en fincas y su relación con la trazabilidad bovina) indica que los registros pecuarios deben de ser ordenados en su almacenamiento de la información, relacionada con el origen, manejo, comportamiento y destino de los animales y cuyo objetivo principal es brindar información ordenada que permita al productor tomar mejores decisiones en relación a la gestión productiva y económica de sus fincas. Se menciona que es una tarea pendiente porque la mayoría de los ganaderos no llevan registros, esto puede deberse a muchos factores, pero me atrevo a afirmar que es porque la mayoría de ellos no le encuentra la importancia de su uso, y por lo tanto, aducen argumentos tales como: Falta de tiempo para realizarlos, no lo ven necesario porque todo el registro lo llevan mentalmente, que no les sirve para nada, entre otros. A pesar de no registrar información, siempre será una gran oportunidad para que los técnicos, tanto privados como lo de todos los programas y proyectos de apoyo a los productores retomen esta tecnología, la que, si es implementada adecuadamente, se convierte en un verdadero beneficio para los ganaderos. Implementar los registros pecuarios no es solamente enseñar al ganadero a anotar información en los formatos. El verdadero éxito está en ayudarles a interpretar, analizar el consolidado de dicha información para que, en la medida del caso, se tomen mejores decisiones para revertir alguna situación que está provocando baja eficiencia en la producción y productividad del ganado. La promoción del uso de herramientas informáticas contribuye mucho a esta tarea. Además, hoy por hoy, los registros pecuarios son parte fundamental de la trazabilidad bovina, ya que los ganaderos deben registrar los movimientos (ingresos y salidas de animales), los nacimientos (fecha del evento, sexo de la cría, tendencia racial) así como las muertes. Ante esto, el tema de la identificación animal (areteo, enchapado) que se promueve a través de la NTON, facilita la toma de información. 


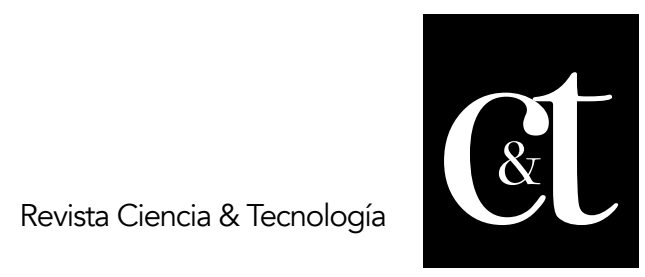

No. 23, 31 de julio de 2019

ISSN impreso: 1390 - 6321

ISSN online: 2661 - 6734

Una vez enchapado el ganado, los datos estarán referidos a los números únicos e irrepetibles de los aretes puestos a cada animal, lo que evitará la confusión entre los nombres comunes y propios con que los ganaderos identifican a sus animales.

\section{Conclusiones}

Una vez hecha la revisan bibliográfica los autores coinciden en que la implementación de los registros productivos es un factor muy importante la producción ganadera, su implementación demanda de un análisis de todo el proceso de la cría de ganado y de los resultados que estos generen durante su vida productiva y reproductiva de cada animal. La implementación de estos registros tiene que ser progresivos y de acuerdo a las etapas de los animales y de las actividades que se den dentro de todo el ciclo productivo, tienen que ser fáciles de llenar y de entender. Así pues, al generar un registro fácil de llenar y de entender ayudará y contribuirá a que los ganaderos se comprometan a llevarlos y cumplirlos. Habituar a los ganaderos que lleven sus registros es uno de los inconvenientes que se tiene ya que en varios casos como se indica en la revisión no llevan porque todo lo llevan mentalmente, pero esta información en ese momento no va a demostrar datos en todo el ciclo productivo entendiendo como un ciclo desde el nacimiento hasta la muerte del animal o desde el inicio hasta la culminación de una actividad. Al final del ciclo es necesario hacer un balance de todo y poder determinar en ese momento si la producción fue rentable o no.

\section{Referencias bibliográficas}

AGROCALIDAD - PEFA. (2017). Proyecto de erradicación de fiebre aftosa 2017. Machala: Ecuador.

Benavides, P. V. (2009). Implementación de un sistema de registros en una finca ganadera". Informe de Práctica de Especialidad presentado como requisito parcial para optar al grado de Bachillerato en Ingeniería Agropecuaria Administrativa con Énfasis en Empresas Agropecuarias. Cartago, Costa Rica, Cartago, Costa Rica, Cartago, Costa Rica.

Correa, C. A. (2015). Implementación de registros productivos y reproductivos en tres unidades ganaderas del departamento de Córdoba. Trabajo de grado para optar al título de zootecnista. Caldas-Antioquia, Caldas-Antioquia, Colombia. elnuevodiario.com.ni. (04 de 03 de 2013). Los registros pecuarios en fincas y su relación con la trazabilidad bovina. Obtenido de

https://www.elnuevodiario.com.ni/economia/279380-registros-pecuarios-fincassurelacion-trazabilida/

Lee Rhinehart. (13 de 10 de 2017). ATTRA es un servicio de información sobre agricultura sustentable a nivel nacional.

Recuperado de https://attra.ncat.org/attrapub/viewhtml.php?id=331 